\title{
Dysfunctional Striatal Systems in Treatment-Resistant Schizophrenia
}

\author{
Thomas P White ${ }^{1,2}$, Rebekah Wigton ${ }^{1,3,4}$, Dan W Joyce', Tracy Collier', Alex Fornito ${ }^{5}$ and \\ Sukhwinder S Shergill*,I
}

' Cognition, Schizophrenia and Imaging Laboratory, Department of Psychosis Studies, Institute of Psychiatry, Psychology and Neuroscience, London, UK; ${ }^{2}$ School of Psychology, University of Birmingham, Birmingham, UK; ${ }^{3}$ Beth Israel Deaconess Medical Center, EEG Lab, Neurology, Boston, MA, USA; ${ }^{4}$ Harvard Medical School, Department of Neurology, Boston, MA, USA; ${ }^{5}$ School of Psychological Sciences and Monash Biomedical Imaging, Monash University, Clayton, VIC, Australia

\begin{abstract}
The prevalence of treatment-resistant schizophrenia points to a discrete illness subtype, but to date its pathophysiologic characteristics are undetermined. Information transfer from ventral to dorsal striatum depends on both striato-cortico-striatal and striato-nigro-striatal subcircuits, yet although the functional integrity of the former appears to track improvement of positive symptoms of schizophrenia, the latter have received little experimental attention in relation to the illness. Here, in a sample of individuals with schizophrenia stratified by treatment resistance and matched controls, functional pathways involving four foci along the striatal axis were assessed to test the hypothesis that treatment-resistant and non-refractory patients would exhibit contrasting patterns of resting striatal connectivity. Compared with non-refractory patients, treatment-resistant individuals exhibited reduced connectivity between ventral striatum and substantia nigra. Furthermore, disturbance to corticostriatal connectivity was more pervasive in treatment-resistant individuals. The occurrence of a more distributed pattern of abnormality may contribute to the failure of medication to treat symptoms in these individuals. This work strongly supports the notion of pathophysiologic divergence between individuals with schizophrenia classified by treatmentresistance criteria.

Neuropsychopharmacology (2016) 4I, 1274-1285; doi:10.1038/npp.2015.277; published online 21 October 20I5
\end{abstract}

\section{INTRODUCTION}

Establishing why current antipsychotic medication fails to assuage hallucinations (aberrant perceptions) or delusions (fixed, false beliefs) in $\sim 30 \%$ of schizophrenia patients (Lieberman et al, 2005) is a key clinical problem and relies on identifying core neural features that predict treatment resistance. Current medication for schizophrenia principally targets the striatum (Seeman and Lee, 1975); and clinical potency is predicted by its binding to and blockade of the dopamine $\mathrm{D}_{2}$ receptor (Creese et al, 1976). However, the observation that responders and treatment-resistant individuals exhibit virtually identical $\mathrm{D}_{2}$ receptor occupancy levels (Wolkin et al, 1989) suggests that occupancy alone is insufficient to produce symptomatic alleviation. More recent observations that treatment-resistant patients differ from responders in terms of both dopamine concentrations in the limbic and associative striatal subdivisions and glutamate concentration in the anterior cingulate cortex

*Correspondence: Professor SS Shergill, Cognition, Schizophrenia and Imaging Laboratory, Department of Psychosis Studies, Institute of Psychiatry, Psychology and Neuroscience, de Crespigny Park, London SE5 8AF, UK, Tel: +44 (0)207 848 0350, Fax: +44 207848 0287, E-mail: sukhi.shergill@kcl.ac.uk

Received 5 June 2015; revised 7 August 2015; accepted 9 August 20I 5; accepted article preview online 9 September 2015
(ACC) (Demjaha et al, 2012, 2014) suggest the presence of discrete pathophysiologic subtypes. Nevertheless, the mechanisms underlying treatment resistance remain incompletely resolved.

Information appears to flow from ventral striatum - where basic stimulus features such as anticipated reward value are encoded-to dorsal structures, where distinct parallel circuits facilitate this transfer, and refine information content for subsequent appropriation by learning and action processes (Botvinick et al, 2009; Croxson et al, 2009; Haber and Knutson, 2010). Striato-cortico-striatal loops predominantly involving prefrontal cortex (PFC) projections have been delineated in nonhuman primates (Alexander et al, 1986), and confirmed in humans with diffusion tensor imaging (Leh et al, 2007; Lehericy et al, 2004) and resting-state functional magnetic resonance imaging (rs-fMRI) (Di Martino et al, 2008). These loops include: a ventral circuit anchored in the inferior limbic subdivision of the striatum and comprising connections with orbitofrontal cortex (OFC), ventromedial PFC, medial thalamus, and limbic regions that is fundamental to associative learning and reward-mediated decision making (Knutson and Cooper, 2005); and a dorsal circuit, including the associative subdivision of the striatum, dorsolateral PFC and mediodorsal and ventroanterior thalamus that maintains information relating to reward outcomes (O'Doherty et al, 
2004). Furthermore, there is convergent evidence for the complementary involvement of these corticostriatal networks in psychotic illness. Compromised ventral circuit function has been well established by consistently reduced activation of ventral striatum and PFC during reward processing in schizophrenia (Heinz and Schlagenhauf, 2010; White et al, 2013), structural changes of ventromedial PFC after or during the transition to a first illness episode (Mechelli et al, 2011), and an upregulation of ventral striatum dopamine concentration in psychotic individuals (Fusar-Poli and Meyer-Lindenberg, 2013). However, preferential elevation of dopamine in dorsal striatum has also been reported in both unmedicated patients and individuals in an at-risk mental state (ARMS) for developing psychosis (Howes et al, 2009; Kegeles et al, 2010).

Subcircuits comprising pathways between striatum and substantia nigra (SN) are less publicized but equally pivotal for information flow through the striatum; playing a seemingly crucial role in instrumental learning and habit formation (Belin and Everitt, 2008). These projections are more broadly distributed than corticostriatal pathways (Haber and Knutson, 2010). Despite this intermingled, clustered arrangement, ventral tegmental area and medial $\mathrm{SN}$ are generally associated with ventral striatum, and central and ventrolateral SN with associative and sensorimotor striatum, respectively (Haber and Fudge, 1997; Haber et al, 2000; Nauta and Domesick, 1978; Somogyi et al, 1981). Although ventral striatum receives sparse SN input, it projects to a large region of midbrain and is therefore a strong modulator of SN activity. In contrast, dorsal striatum (caudate/putamen) receives diverse and numerous afferent connections from SN, and is therefore heavily influenced by $\mathrm{SN}$, but itself extends limited reciprocal projections. Individuals with schizophrenia have been recently shown to exhibit reduced nigrostriatal connectivity (Yoon et al, 2013, 2014), but striato-nigro-striatal connections have not yet been investigated in relation to treatment resistance.

Studying functional connectivity (FC) in striatal circuits at rest circumvents issues of performance (eg, intersubject differences and practice and ceiling/floor effects) that confound task-based functional imaging investigations. As yet, no robust structural or functional brain correlates have been associated specifically with treatment-resistant schizophrenia (Nakajima et al, 2015). Investigation of multiple corticostriatal circuits has, however, revealed complex, subtle alterations in association with both vulnerability to psychosis and clinical features of the disorder. ARMS individuals display hypoconnectivity (as compared with control subjects) in the circuit involving dorsal caudate, right DLPFC, medial PFC, and thalamus, but hyperconnectivity between ventral putamen, frontoinsular cortex, and superior temporal gyrus (Dandash et al, 2014). Similarly, in individuals with first-episode psychosis (FEP) and their first-degree relatives, functional connectivity is enhanced for the ventral circuit and reduced for the dorsal circuit (Fornito et al, 2013). These findings, together with the assumed importance of striatal networks for treatment response and the growing evidence for a dissociable neurophysiologic foundation for treatmentresistant schizophrenia, guided our interest in clarifying whether treatment-resistant individuals are differentiable from other individuals with schizophrenia on the basis of their striatal connectivity.
If pharmacological blockade of striatal $\mathrm{D}_{2 / 3}$ receptors effects clinical improvement and normalization of brain activity in some patients but not others, it is likely that treatment-responsive and -resistant patients differ in terms of their striatal network function. A recent study has used this idea to examine prospective treatment response, identifying changes in striatal connectivity with prefrontal and limbic regions as important in symptomatic alleviation (Sarpal et al, 2015). Guided by these observations, the rationale that treatment-resistant individuals would differ from non-refractory patients in these brain substrates that track clinical improvement, and the idea that treatmentresistant individuals would exhibit striatal FC abnormalities indicative of their specific cognitive and behavioral impairments, we addressed two principal hypotheses. First, treatment-resistant individuals with schizophrenia would display reduced connectivity along nigrostriatal pathways compared with non-refractory individuals, as learning and its influence on action can be impaired in treatment-resistant individuals (Dratcu et al, 2007; Kolakowska et al, 1985), and related processes are regulated by striatum and substantia nigra (Braver et al, 1999a; Braver and Cohen, 1999b; D'Ardenne et al, 2012). Second, frontostriatal disruptions observed when comparing patients with healthy individuals (Quide et al, 2013; Sarpal et al, 2015) would differ as a function of treatment resistance. In addition, as the persistence of positive symptoms is fundamental to treatment resistance, and with the aim of building on previous FEP observations (Fornito et al, 2013), we assessed the extent to which current positive symptom severity predicted striatal FC in treatment-resistant and non-refractory patients.

\section{MATERIALS AND METHODS}

\section{Participants}

Thirty-eight right-handed individuals satisfying DSM-IV criteria for schizophrenia took part in this fMRI study. These individuals were stratified according to their documented response to antipsychotic treatment in electronic medical records: 16 met modified Kane criteria for treatment-resistant schizophrenia on the basis of: (1) completion of at least two sequential 4-week antipsychotic trials at a daily dose of $400-600 \mathrm{mg}$ chlorpromazine (or equivalent); (2) persistent psychotic symptoms of at least moderate severity (as indexed by Positive and Negative Syndrome Scale (PANSS) scores (Kay et al, 1987) on one or more positive subscale measure); and (3) impaired occupational functioning (as indexed by a score of $\leq 59$ on the Global Assessment of Function (Conley and Kelly, 2001; Demjaha et al, 2012)). Patients not satisfying all treatment resistance-related criteria were assigned to the non-refractory schizophrenia group. Medication compliance was assessed by review of pharmacy and medical records. This recruitment strategy, in contrast to the selection of treatmentresistant and treatment-responsive patients (with alleviated symptoms), permitted between patient-group matching in terms of current symptom severity, presenting the capability to dissociate effects of treatment resistance from those of current illness state. Patient groups were group matched for age, sex, and parental socioeconomic status (Rose and Pevalin, 2001) with each other and a sample of 20 healthy 
Table I Sample Demographic and Clinical Characteristics

\begin{tabular}{|c|c|c|c|c|}
\hline Variable & $\begin{array}{l}\text { Treatment-resistant } \\
\text { schizophrenia }(n=16)\end{array}$ & $\begin{array}{c}\text { Non-refractory } \\
\text { schizophrenia }(n=22)\end{array}$ & $\begin{array}{l}\text { Healthy } \\
(n=20)\end{array}$ & $\begin{array}{l}\text { Between-group } \\
\text { comparisons }\end{array}$ \\
\hline Age (years) & $36.69(7.86)$ & $37.55(9.60)$ & $36.30(9.38)$ & $P>0.5$ for all comparisons \\
\hline $\begin{array}{l}\text { Parental socioeconomic } \\
\text { status (NS-SEC) }\end{array}$ & $2.69(1.49)$ & $2.64(1.65)$ & $2.35(1.59)$ & $P>0.5$ for all comparisons \\
\hline Positive PANSS & $17.88(5.90)$ & I5.32 (3.87) & & $\begin{array}{c}\text { TR vs NR: } T(36)=1.61 \\
P=0.115\end{array}$ \\
\hline Age at onset of illness (years) & $21.34(4.40)$ & $25.57(5.88)$ & & 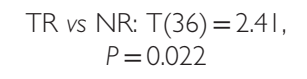 \\
\hline Duration of illness (years) & $15.47(6.4 \mid)$ & $11.86(10.35)$ & & $\begin{array}{c}\text { TR vs NR: } T(33.79)=1.30 \\
P=0.201\end{array}$ \\
\hline $\begin{array}{l}\text { Current antipsychotic } \\
\text { medication }\end{array}$ & $\begin{array}{l}\text { Clozapine }(n=11) \text {, Aripiprazole }(n=2) \text {, } \\
\text { Olanzapine }(n=2) \text {, Amisulpride }(n=1) \text {, } \\
\text { Haloperidol }(n=1) \text {, Paliperidone }(n=1) \text {, } \\
\text { Quetiapine }(n=1) \text {, Zuclopenthixol }(n=1)\end{array}$ & $\begin{array}{c}\text { Olanzapine }(n=9) \text {, Aripiprazole }(n=3) \text {, } \\
\text { Risperidone }(n=3) \text {, Amisulpride }(n=1) \text {, } \\
\text { Fluphenazine decanoate }(n=1) \text {, Haloperidol } \\
(n=1) \text {, Pipotiazine }(n=1) \text {, Quetiapine } \\
(n=1) \text {, Venlafaxine }(n=1) \text {, Zuclopenthixol } \\
(n=1)\end{array}$ & & \\
\hline $\begin{array}{l}\text { Antipsychotic medication } \\
\text { dosage (mg/day; CPZ) }\end{array}$ & $764.06(339.15)$ & $281.68(299.14)$ & & $\begin{array}{c}\text { TR vs NR: } T(36)=4.64 \\
\quad P=5 \times 10^{-4}\end{array}$ \\
\hline
\end{tabular}

Abbreviations: CPZ, chlorpromazine equivalent; HC, healthy controls; NR, non-refractory schizophrenia; NS-SEC, National Statistics Socio-Economic Classification (Rose and Pevalin, 200 I); PANSS, Positive and Negative Syndrome Scale for schizophrenia (Kay et al, 1987); TR, treatment-resistant schizophrenia; WASI, Wechsler Abbreviated Scale of Intelligence (Wechsler, 1999).

Bracketed values denote SDs.

volunteers. Healthy participants were recruited by local poster advertisement. Respondents were excluded from study if: they reported a personal history of psychiatric or neurological illness; a recent history of illicit substance use; or a history of psychotic illness in a first-degree relative; or exhibited a major current physical illness. Details of these participants' demographics and clinical characteristics are presented in Table 1. Ethical approval was provided by Central London Research and Ethics Committee 3. All participants provided informed written consent and were given a monetary inconvenience allowance for participation.

\section{Design}

All patients participated in one MRI session and experienced no amendment to their ongoing antipsychotic treatment regimen.

\section{fMRI Data Acquisition}

The fMRI data for each scanning session comprised 300 gradient-echo echo-planar images (TR/TE: 2000/30 ms, flip angle: $75^{\circ}$, matrix: $\left.64 \times 64\right)$ acquired on a 3 -Tesla GE Signa MR scanner (GE Healthcare) at the Institute of Psychiatry, London. Each whole-brain image contained 37 noncontiguous slices of $2.4 \mathrm{~mm}$ thickness separated by a distance of $1 \mathrm{~mm}$, and with in-plane isotropic voxel resolution of $3.4 \mathrm{~mm}$. Participants were instructed to remain still with gaze fixed on a central cross for the duration of this 10-min resting-state scan. A high-resolution T1-weighted structural scan was acquired for each participant using a fast-spoiled gradient-echo pulse sequence (repetition time $=9.4 \mathrm{~ms}$, echo time $=3.8 \mathrm{~ms}$, flip angle $=12^{\circ}$, time to inversion $=450 \mathrm{~ms}$ ).

\section{fMRI Analysis}

The fMRI data were preprocessed using SPM8 (Statistical Parametric Mapping, Wellcome Department of Imaging Neuroscience, University of London, UK). Data were slice-time corrected and realigned to the first image of each series, normalized via unified segmentation of subjectspecific anatomical data coregistered to the SPM-T1 
template, and smoothed using a 6-mm full-width at half maximum Gaussian kernel. Segmented white matter (WM) and cerebrospinal fluid (CSF) images were thresholded at $50 \%$ tissue probability and binarized to create nuisance variable masks.

To facilitate potential comparisons with recent related findings, further processing and seed definition followed procedures outlined elsewhere (Dandash et al, 2014; Fornito et al, 2013). Seeds were defined in both hemispheres as $3.5 \mathrm{~mm}$ radius spheres at the following stereotaxic coordinates: dorsal caudate ( $\mathrm{DC} ; \mathrm{x}= \pm 13, \mathrm{y}=15, \mathrm{z}=9$ ); ventral striatum/nucleus accumbens (VS; $x= \pm 9, y=9, z=-8$ ); dorsal-caudal putamen ( $\mathrm{dcP} ; \mathrm{x}= \pm 28, \mathrm{y}=1, \mathrm{z}=3$ ); and ventral-rostral putamen (vrP; $\mathrm{x}= \pm 20, \mathrm{y}=12, \mathrm{z}=-3$ ) (Dandash et al, 2014). To complement the original investigation of $\mathrm{Di}$ Martino et al (2008), effects were additionally modeled in relation to their remaining two seeds, but as per previous work (Dandash et al, 2014), experimental focus was placed upon the former four seeds. Component-based correction (CompCor) of temporal confounds relating to head movement and physiological noise was performed using the CONN toolbox (v.14) (Whitfield-Gabrieli and Nieto-Castanon, 2012). Under the rationale that related noise effects are not spatially uniform, and that regional signals encode temporally distinct linear combinations of them, CompCor parses signals measured within specified masks into linearly additive temporal components whose effects on connectivity metrics can all be mitigated. Accordingly, the first 5 principal components of the WM- and CSF-mask signals were calculated, and the first eigenvariate of activity within each of the 6 bilateral seeds was estimated after regressing out linear effects of the 6 realignment parameters, their first derivatives, and the 10 noise components. Preprocessed data were temporally bandpass filtered $(0.01-0.1 \mathrm{~Hz})$.

First-level FC analyses were performed using general linear models, as implemented in SPM8. These modeled individual-specific covariation between the activity of each seed and the rest of the brain, and comprised regressors for the 6 seed regions' time courses, 6 realignment parameters and their first derivatives, and the 10 noise components. Second-level models were estimated according to our explicit hypotheses. First, to test whether FC between each striatal seed and the rest of the brain differed between individuals with treatment-resistant and non-refractory schizophrenia, independent-samples $t$-tests were conducted for these groups for each seed. To permit dissociation of effects relating to, and those independent from, the severity of current psychotic illness, covariates included the positive, negative, and general PANSS subscores. To further account for potential motion effects on connectivity estimates, the effects of four summary measures of head movement were added as covariates (Fornito et al, 2013; Van Dijk et al, 2012) in these and all subsequent between-group, second-level analyses (further details regarding the calculation of head movements and their comparison across study groups can be found in Supplementary Materials and Methods). Second, to examine potential idiosyncrasies in connectivity specific to each patient group, independent samples $t$-tests were conducted to compare their whole-brain connectivity patterns with those of the healthy individuals. Third, to evaluate patient-groupspecific relationships between current schizophrenic symptomatology and whole-brain striatal FC, analysis of covariance (ANCOVA) models were estimated for each of the four seeds, independently for treatment-resistant and nonrefractory schizophrenia. These models included the three PANSS subscores as predictors and the four summary measures of head movement and CPZ dosage as covariates. The inclusion of negative and general symptom subscores allowed detection of effects specific to the positive symptom subscore.

Finally, ANCOVA tests were used to investigate the extent to which striatal FC was predicted by antipsychotic medication dosage in individuals with schizophrenia. Effects were assessed via within-group models because, as shown in Table 1, there was a significant difference in CPZ dosages between the patient groups. Covarying out the effects of variables that differ between groups does not statistically equate to conducting the same experiment in individuals matched on that variable, as has been elegantly and repeatedly discussed elsewhere (see, eg, Suckling, 2011). With this in mind, independent ANCOVA tests were run for the treatment-resistant and non-refractory individuals with schizophrenia. The effects of medication dosage on each seed's functional connectivity were examined separately in models incorporating subject-specific contrast images for the functional connectivity of that seed and covarying out effects of the three PANSS subscores and the four summary measures of head movement. Importantly, on account of the betweengroup differences in $\mathrm{CPZ}$ dosage, the covariates were not mean centered. This ensured that the effects of each particular dosage were consistently described across both groups.

For all analyses significance was ascribed according to a family-wise error corrected cluster-wise threshold determined using the AlphaSim permutation procedure implemented in the REST toolbox (http://pub.restfmri.net) in a manner identical to previous investigations of striatal FC in psychosis (Dandash et al, 2014; Fornito et al, 2013). To reduce type 1 error, the conventional family-wise error corrected cluster threshold of 0.05 was further Bonferroni corrected to reflect the number of seeds tested. Significant between-patient group effects are reported only in those voxels judged significant in the healthy individuals. Seedspecific masks were constructed from the results of onesample $t$-tests conducted on the healthy group contrast images, covarying for effects of the summary measures of head movement, and using the cluster-level significance criterion described above (Supplementary Figure S1). These were selected under the rationale that they represent the core connectivity circuit for each seed. Supplementary Figures S2 and S3 illustrate overlap between healthy group findings and significant clusters in non-refractory and treatment-resistant schizophrenia respectively.

\section{RESULTS}

\section{Divergent Functional Connectivity in Treatment- Resistant and non-refractory Schizophrenia}

In comparison with healthy individuals, the patient groups exhibited divergent patterns of corticostriatal abnormality. The treatment-resistant patients displayed reduced FC between VS and middle frontal gyrus, between DC and sensorimotor cortex, and in terms of striato-striatal 


\section{Healthy Individuals > Non-refractory Schizophrenia}
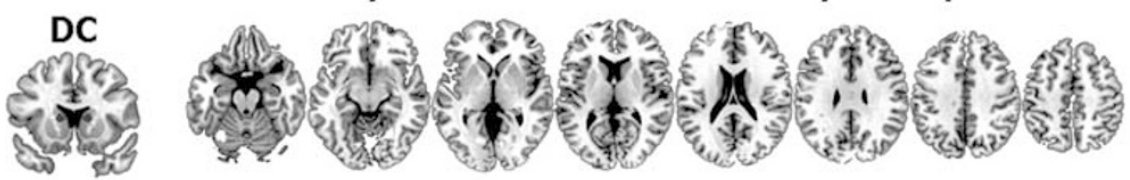

VS

Healthy Individuals > Treatment-resistant Schizophrenia
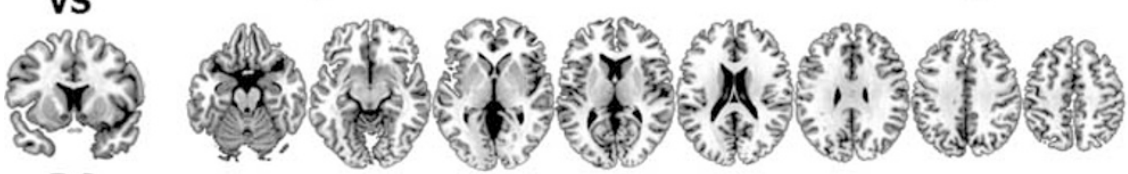

DC
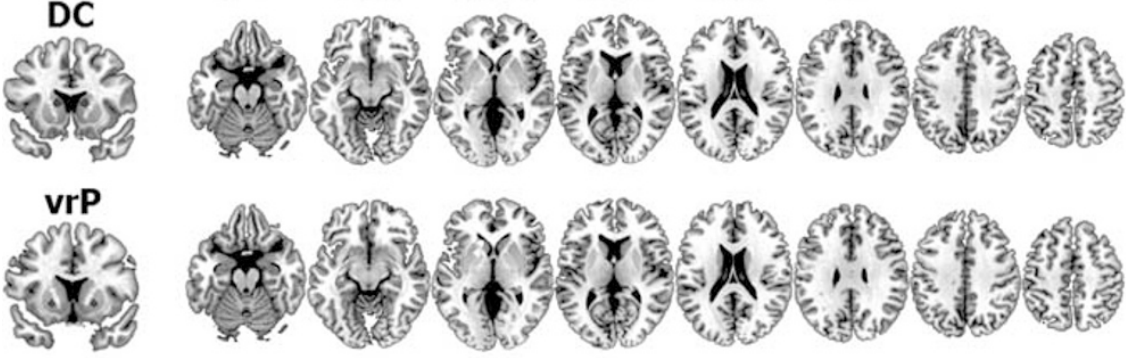

2 7

Figure I Specific disease-related reductions in striatal connectivity, showing: reductions in resting functional connectivity (FC) between the dorsal caudate (DC) seed and prefrontal cortex (top row); reductions in FC between the ventral striatum (VS) and middle frontal gyrus (second row); reductions in FC between DC and sensorimotor cortex (third row); and reductions in FC between ventrorostral putamen (vrP) and proximal structures of the striatum (bottom row). All results are thresholded at a family-wise error corrected cluster threshold of 0.0125 (to correct for the investigation of four seeds) and overlaid on a standardized TI-weighted template image. A full color version of this figure is available at the Neuropsychopharmacology journal online.

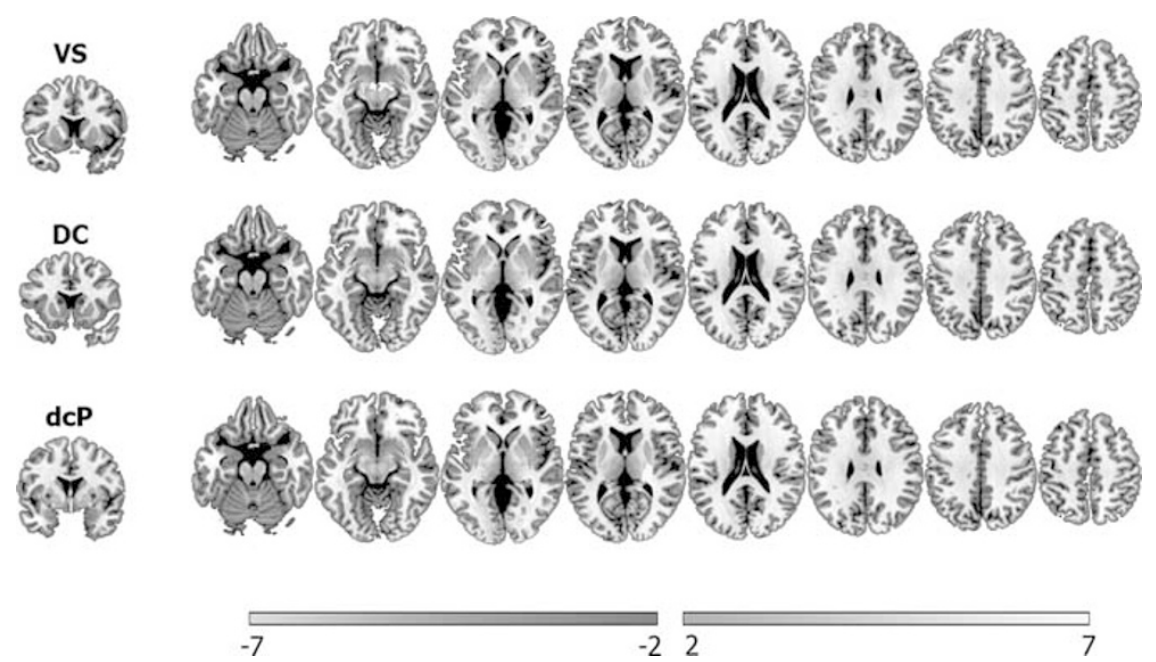

Figure 2 Significant differences in striatal connectivity between treatment-resistant and non-refractory individuals with schizophrenia, controlling for effects of current psychiatric symptom severity and movement. Left column shows the seeds for which the subsequent results within each row apply. Blue scale denotes regions significantly less connected in non-refractory patients as compared with the treatment-resistant individuals, and yellow scale denotes regions significantly more connected with seed in non-refractory individuals. All results are thresholded at a family-wise error corrected cluster threshold of 0.0125 (to correct for the investigation of four seeds) and overlaid on a standardized TI-weighted template image. DC, dorsal caudate; dcP, dorsocaudal putamen; VS, ventral striatum. A full color version of this figure is available at the Neuropsychopharmacology joumal online.

connectivity of circuits involving the vrP seed (Figure 1 and Table 2). In contrast, significantly reduced functional connectivity was found between the DC and rostral PFC extending into dorsolateral PFC, and DC and visual cortex in non-refractory patients as compared with healthy controls (Figure 1 and Table 2). Between-group comparisons for the other seeds produced nonsignificant results. Compared with non-refractory patients, treatment-resistant individuals with schizophrenia exhibited reduced striatonigral FC between VS and substantia nigra, and reduced FC between $\mathrm{dcP}$ and the pulvinar of the thalamus. In addition, they exhibited enhanced functional connectivity between DC and medial 
Table 2 Significant Gray Matter Foci of Between-Group Differences in Striatal Resting-State Functional Connectivity

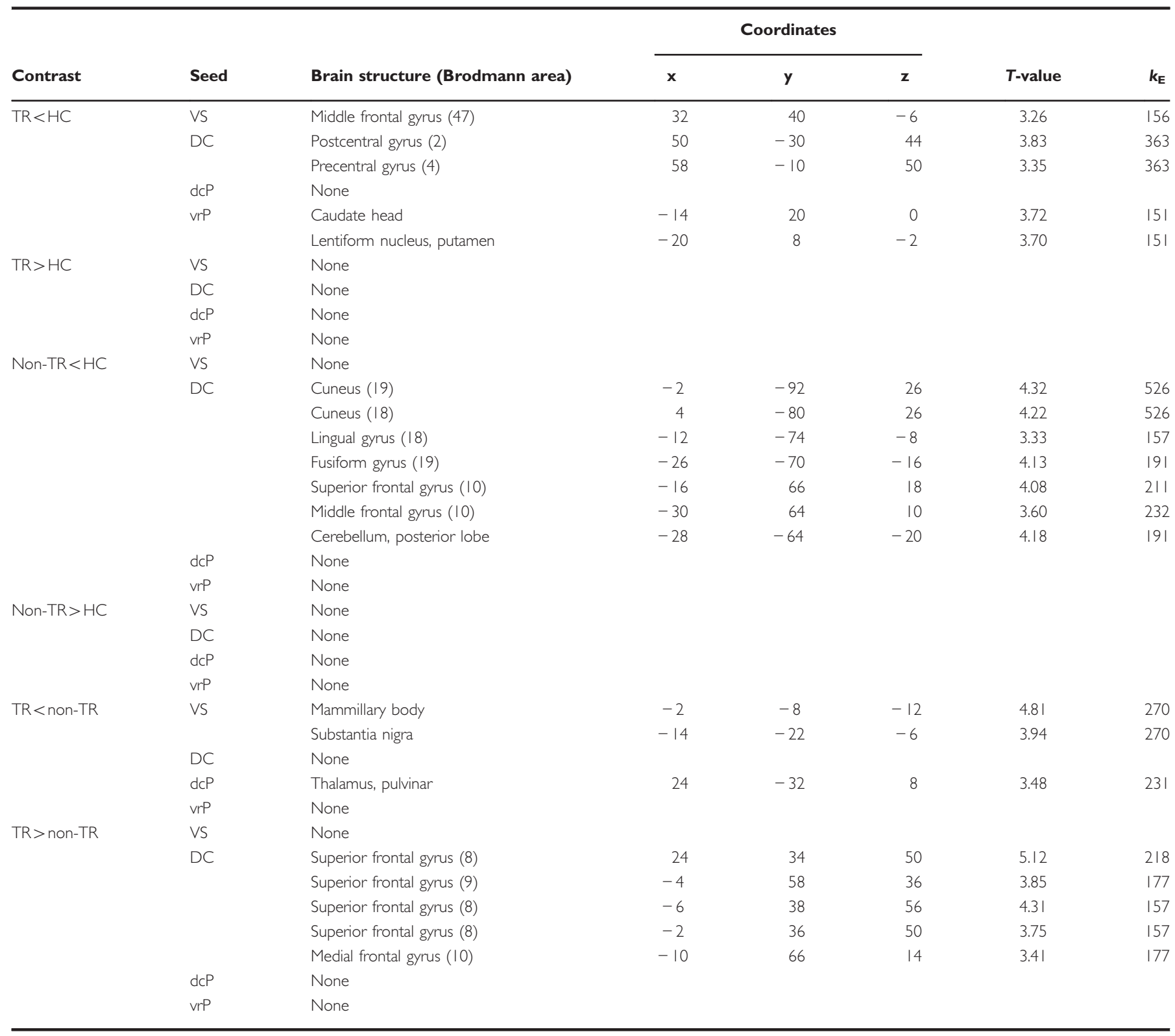

Abbreviations: DC, dorsal caudate; dcP, dorsocaudal putamen; HC, healthy controls; TR, treatment-resistant schizophrenia; vrP, ventrorostral putamen; VS, ventral striatum.

and superior PFC compared with non-refractory individuals (Figure 2 and Table 2).

\section{Relationships between FC and Positive Symptoms of Schizophrenia}

In treatment-resistant schizophrenia, increased positive PANSS subscore was associated with reduced functional connectivity between VS and parietal midline structures and middle frontal gyrus. In the same group, increased positive PANSS subscore was also associated with increased FC between dorsal striatum seeds and regions including precuneus, posterior cingulate, and medial prefrontal cortex. In non-refractory schizophrenia, positive PANSS subscore was positively associated with FC between VS and anterior cerebellum (Figure 3 and Table 3 ). No other relationships between positive PANSS score and functional connectivity were significant.

\section{Relationships between FC and Antipsychotic Dosage}

In treatment-resistant schizophrenia, CPZ dosage significantly positively predicted FC of the striatum with several cortical regions, including lingual gyrus (Table 4). Significant inverse relationships between medication and striatal FC were limited to the findings relating to the VS seed, whereby medication inversely predicted connectivity with regions including posterior cingulate gyrus, lingual gyrus, cerebellum, and prefrontal cortex (Table 4).

In contrast, for non-refractory individuals with schizophrenia, no significant positive associations between $\mathrm{CPZ}$ dosage and FC were observed for any of the striatal regions 


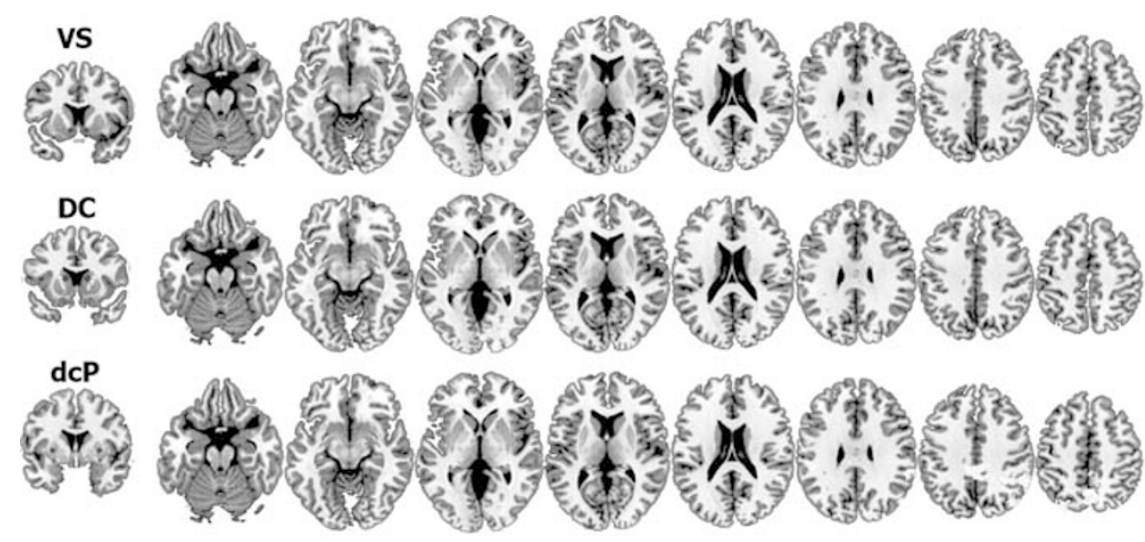

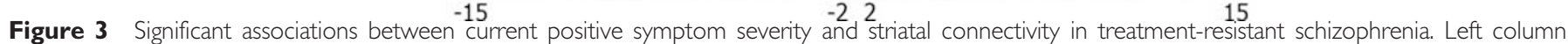

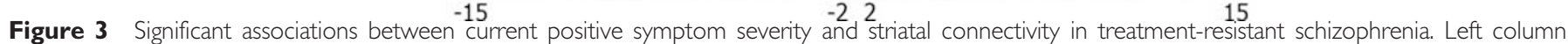

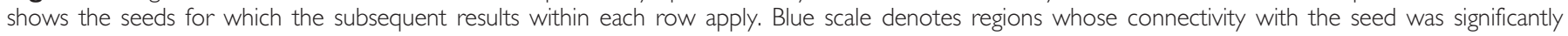

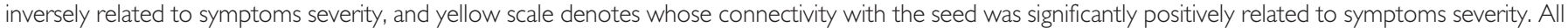

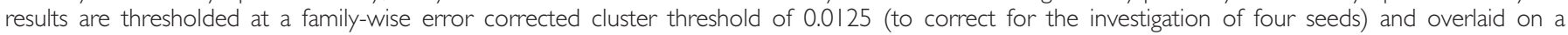

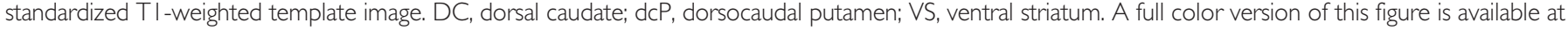
the Neuropsychopharmacology journal online.

investigated. However, CPZ dosage inversely predicted FC with prefrontal cortex for the $\mathrm{DC}$, dcP, and $\operatorname{vrP}$ seeds (Table 4).

\section{DISCUSSION}

Reducing the persistent symptoms of treatment-resistant schizophrenia is contingent on understanding their neurophysiologic provenance. There is evidence that treatmentresistant individuals differ from responsive patients in terms of striatal dopamine synthesis capacity and prefrontal glutamate availability (Demjaha et al, 2012, 2014). In view of these findings, this study investigated functional connectivity with a focus on these brain structures in individuals with schizophrenia stratified by treatment resistance and healthy control subjects. It identifies potential idiosyncrasies of treatment-resistant schizophrenia on two principal fronts. First, patients with schizophrenia with treatment resistance exhibited reduced connectivity between VS and SN; reduced connectivity between the $\mathrm{dcP}$ and thalamus; and elevated connectivity between DC and medial PFC (Figure 2 and Table 2). We thereby identify diminished cross-talk between VS and $\mathrm{SN}$ as a potential mechanism for treatment resistance. In light of the relative abundance of connections from VS to SN (Haber and Knutson, 2010), it is likely that this finding represents a diminution of the influence of VS on other brain structures in treatment-resistant schizophrenia.

The current data also emphasize specific corticostriatal pathways along which information flow within reward systems involving cortex and basal ganglia differ between these patient groups. Specifically, coupling between DC and superior and medial prefrontal cortex is reduced in nonrefractory compared with treatment-resistant patients (Figure 2 and Table 2). These notable differences at multiple sites along the putative ventral-dorsal transfer axis suggest that there may be differential dysfunction affecting the feedback systems that process and integrate reward-related information with cognition and action. Second, as compared with healthy control subjects, the schizophrenia groups displayed varying differences in striatal FC that were for the most part demonstrative of reduced corticostriatal and striato-striatal connectivity in patients. Treatment-resistant individuals exhibited reduced connectivity of the VS with orbitofrontal cortex, between DC and sensorimotor regions, and between vrP and a striatal cluster encompassing caudate head and putamen. In contrast, significant differences in striatal FC in non-refractory patients were limited to the DC, whose connectivity with regions including rostrolateral PFC, occipital cortex, and cerebellum was attenuated (Figure 2). These findings imply that corticostriatal dysconnectivity is more anatomically distributed in treatment-resistant individuals, and this could in part explain the reduced efficacy of medication in these individuals. However, neuroimaging data in treatment-resistant schizophrenia reported to date, which have failed to uncover robust correlates of poor response to medication (Nakajima et al, 2015), do not support this interpretation.

This work upholds and adds to recent observations from FEP and ARMS cohorts of hypoconnectivity (as compared with controls) of dorsal corticostriatal circuits (Dandash et al, 2014; Fornito et al, 2013). However, in our study, reduced functional connectivity (compared with controls) between DC and PFC was specific to non-refractory patients. Furthermore, treatment-resistant individuals displayed elevated connectivity between DC and medial PFC when compared with non-refractory patients, suggesting that frontostriatal hypoconnectivity is a less useful disease marker for treatment-resistant individuals; providing a further point of neurophysiologic distinction between patients stratified by response.

Contrary to previous investigations, we found little evidence for ventral corticostriatal hyperconnectivity in schizophrenia. However, although ventral circuit hyperconnectivity provides an elegant candidate mechanism for cognitive features of psychotic illness relating to aberrant salience attribution (Kapur et al, 2005), its empirical support is presently equivocal. In fact, there are numerous reports of 
Table 3 Relationships Between Positive PANSS Subscore and Striatal Resting-State Functional Connectivity in Treatment-Resistant and Nonresistant Schizophrenia

\begin{tabular}{|c|c|c|c|c|c|c|c|c|}
\hline \multirow[b]{2}{*}{ Group } & \multirow[b]{2}{*}{ Seed } & \multirow[b]{2}{*}{ Direction } & \multirow[b]{2}{*}{ Brain structure (Brodmann area) } & \multicolumn{3}{|c|}{ Coordinates } & \multirow[b]{2}{*}{$T$-value } & \multirow[b]{2}{*}{$k_{E}$} \\
\hline & & & & $\mathbf{x}$ & y & $\mathbf{z}$ & & \\
\hline \multirow{12}{*}{ TR } & & Inverse & Cingulate cortex (31) & -10 & -48 & 42 & 3.52 & 172 \\
\hline & & & Precuneus (7) & -8 & -58 & 48 & 3.51 & 172 \\
\hline & & & Middle frontal gyrus (9) & 34 & 26 & 38 & 4.59 & 162 \\
\hline & & & Medial frontal gyrus (6) & 8 & -30 & 64 & 7.18 & 262 \\
\hline & & & Medial frontal gyrus (6) & -2 & -24 & 56 & 5.09 & 262 \\
\hline & & & Middle temporal gyrus (39) & -46 & -70 & 8 & 5.25 & 285 \\
\hline & dcP & Positive & Precuneus (7) & 6 & -68 & 48 & 14.10 & 328 \\
\hline & & & Precuneus (7) & -8 & -62 & 46 & 7.94 & 328 \\
\hline & & & Superior parietal lobule (7) & 38 & -72 & 44 & 5.13 & 260 \\
\hline & & Inverse & None & & & & & \\
\hline & vrP & Positive & None & & & & & \\
\hline & & Inverse & None & & & & & \\
\hline \multirow[t]{7}{*}{ Non-TR } & VS & Positive & Cerebellum, anterior lobe & 14 & -28 & 20 & 5.10 & 156 \\
\hline & & Inverse & None & & & & & \\
\hline & DC & Positive & None & & & & & \\
\hline & & Inverse & None & & & & & \\
\hline & dcP & Positive & None & & & & & \\
\hline & & Inverse & None & & & & & \\
\hline & vrP & Positive & None & & & & & \\
\hline
\end{tabular}

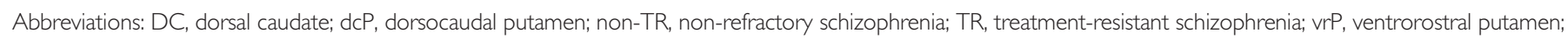
VS, ventral striatum.

reduced functional connectivity of ventral circuit PFC regions in schizophrenia (Backasch et al, 2014; Diaconescu et al, 2011; He et al, 2013; Lynall et al, 2010; Tomasi and Volkow, 2014). Although these discrepant findings may be attributable to factors including clinical heterogeneity, a more complete understanding of schizophrenia-related abnormalities in these networks can be assisted by examining reported effects in the context of a more comprehensive characterization of the individuals involved (Insel, 2014), and with reference to specific abnormalities in task-related behavior.

One of the fundamental cornerstones of clinical practice is to increase the dosage of medication following insufficient clinical improvement in patient symptoms. This can be observed clearly in our sample, where the prescribed medication dose in the treatment-resistant group exceeds that of the non-refractory group (Table 1). However, it does pose a confound for the investigation of treatment-resistant schizophrenia. Covarying out effects of medication dosage across groups is not a valid solution-this would not equate to measuring FC in groups matched for medication dosage-as has been elegantly argued elsewhere (Suckling, 2011). As such, the extent to which the reported betweengroup differences are purely pathophysiological or the result of pharmacological confounds cannot be definitively detailed, and this limits the current findings. Nevertheless, the analyses of the relationships between CPZ and striatal FC imply that the between-group differences were not solely attributable to differences in current medication level. For the most part the observed regional drug effects did not exhibit spatial correspondence with the regions whose FC was found to differ between the patient groups or between the healthy and patient groups. For instance, the most robust associations were noted between dosage and striato-occipital connections in the treatment-resistant group. However, it is noteworthy that medication was inversely related with connectivity between DC and postcentral gyrus in treatment-resistant individuals and that connectivity between DC and an anatomically proximal region of postcentral gyrus was reduced in treatment-resistant individuals as compared with controls, suggesting that this may be a pharmacologically driven between-group effect rather than a direct effect of the disorder. In general, there were more widespread significant dosage-related effects in treatmentresistant schizophrenia. This provides an indication that these individuals are to some extent neurophysiologically responsive to antipsychotic medication despite its failure to treat their symptoms. 
Table 4 Regions in which Striatal Resting-State Functional Connectivity was Significantly Related to Antipsychotic Dosage

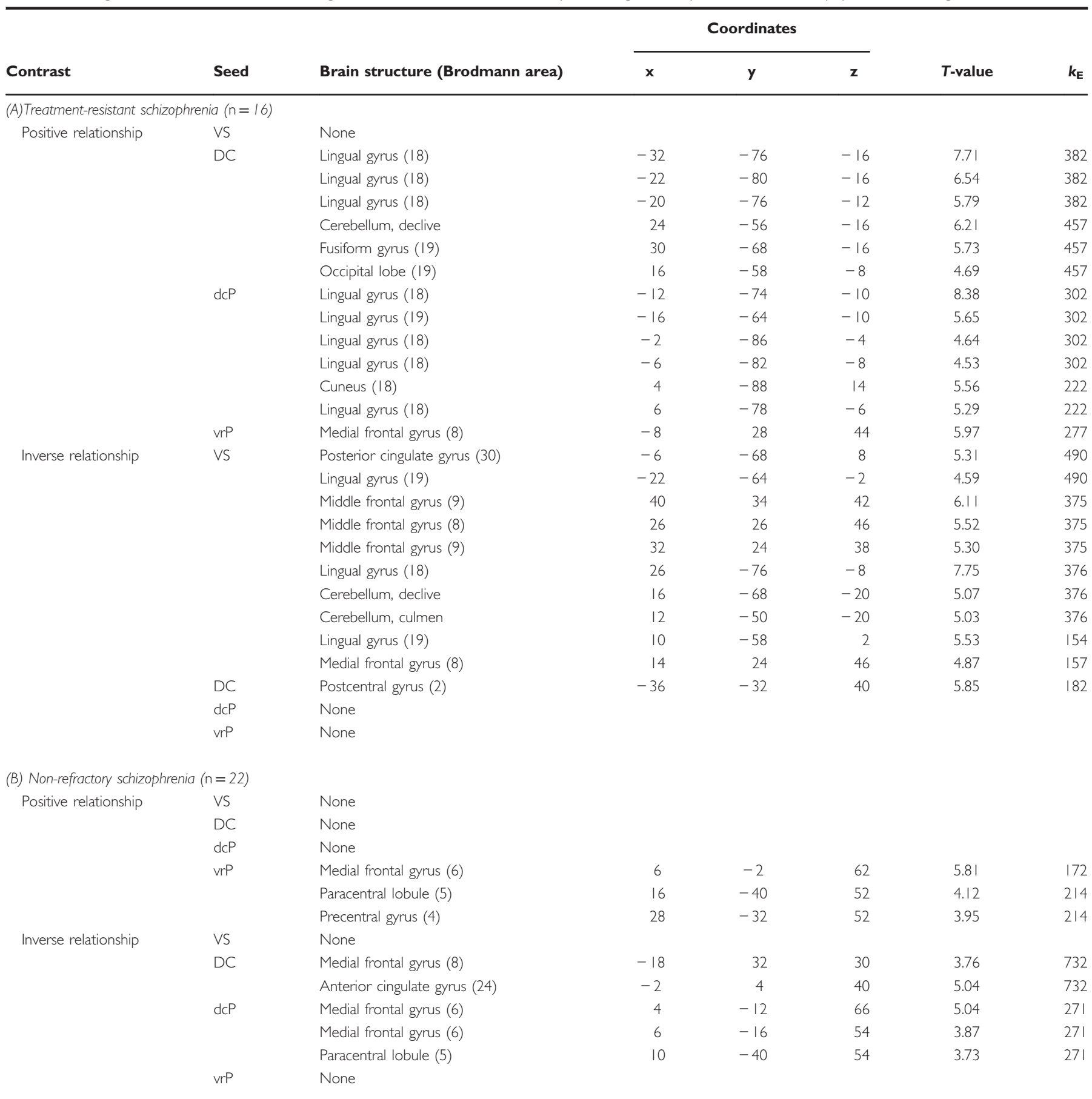

Abbreviations: DC, dorsal caudate; dcP, dorsocaudal putamen; vrP, ventrorostral putamen; VS, ventral striatum.

Further support for the notion that the current effects are not purely medication derived is provided by the regional disparity between the current between-patient group FC effects and recent observations in responsive patients following medication (Sarpal et al, 2015). However, an incomplete understanding of the consequences of long-term antipsychotic treatment and interindividual variation in related phenomena limits this work. Similarly, the possibility that medication with clozapine influenced connectivity in the treatment-resistant group cannot be wholly discounted. Investigating the direct effects of current clozapine treatment on striatal connectivity, and connectome differences specific to individuals with persistent positive symptoms despite clozapine treatment (termed 'ultra-resistant psychosis'), are hugely pertinent areas for future work. Unfortunately, the size of the current subsamples precluded their optimal investigation with this data set (exploratory analyses of clozapine effects are nevertheless presented in 
Supplementary Materials and Methods). However, one benefit of having a majority $(n=11)$ of clozapine-treated patients in the treatment-resistant group is that their treatment is accompanied by monitoring of serum blood levels to ensure that minimum therapeutic levels are achieved during dose titration. This provides a metric for antipsychotic dosage compliance that can otherwise be a concern in these patients.

Previous inverse associations between positive PANSS subscores and striatal functional connectivity (Fornito et al, 2013) were not replicated. In the non-refractory patient group, no significant negative relationships were found. The treatment-resistant individuals did, however, exhibit significant negative relationship between positive symptom severity and connectivity between the VS seed and the cingulate and middle frontal gyrus. In this latter group, those individuals with more severe positive symptoms also displayed enhanced connectivity between $\mathrm{dcP}$ and expansive regions including precuneus, cingulate gyrus, and superior parietal lobule, and between DC and cuneus, medial frontal, and middle temporal gyrus. It would be speculative to suggest on the basis of these relationships that the mechanistic foundations of positive symptoms differ between these patient groups; the observed relationships may, however, represent mechanisms responsible for prolonging these disease features. Particularly noteworthy are the observations of enhanced connectivity between posterior cingulate gyrus, precuneus, and inferior parietal lobule-core regions of the default mode network-and $\mathrm{dcP}$ in the treatment-resistant group that intimate aberrant interconnections between processes of salience attribution and internal monitoring (Fox et al, 2005; Kapur et al, 2005; Raichle et al, 2001).

Although it is advantageous to detect discrepant brain system features stratified on the basis of past treatment response-as this can potentially improve our understanding of the neural basis of treatment resistance-predicting which individuals are unlikely to respond before chronic ineffective medication is of greater import. Long-term medication has been suggested to produce $\mathrm{D}_{2}$ receptor upregulation and associated supersensitivity to dopamine (Ginovart et al, 2009; Samaha et al, 2007), in turn reducing the effective potential of subsequent treatments. Furthermore, ineffective treatment adds to the functional and social incapacitation of long-term illness. As such, tracking the occurrence and timing of differences of cerebral structure and function, such as those currently identified, from the early stages of illness may help identify individuals unlikely to respond to treatment. Future longitudinal work geared toward establishing whether the detected features are a primary component of a treatmentresistant illness subtype or a secondary feature of long-term illness or ineffective pharmacologic treatment is warranted. The current study provides evidence of physiologic substrates related to treatment resistance in schizophrenia; however, although it is likely that both genetic (Frank et al, 2015) and environmental (Hassan and De Luca, 2015) factors underlie treatment resistance and its associated mechanisms, the causative factors remain inadequately explained. Characterizing the treatment-resistance phenotype in terms of clinical and cognitive features has the potential to improve our understanding of the key etiological determinants (Gonzalez-Rodriguez et al, 2014).
By finding that striatal functional connectivity selectively differs between treatment-resistant and non-refractory patients, and that these differences cannot be directly attributable to symptomatic severity at the time of study, this work advocates the notion that contrasting medication response reflects divergent pathophysiologic mechanisms in these individuals. More specifically, variations in corticostriatal association are seen in relation to treatment response in line with recent related findings (Sarpal et al, 2015); and striatonigral dysconnection is identified as a distinct feature of treatment-resistant illness. There is increasing interest in examining glutamatergic treatment options in schizophrenia (Papanastasiou et al, 2013) and elevated glutamate function has been observed in association with treatment resistance (Demjaha et al, 2014). Given recent accounts that the $\mathrm{N}$-methyl-D-aspartate receptor antagonist, ketamine-which disinhibits glutamatergic stimulation of non-NMDA receptors (Moghaddam et al, 1997) - modulates functional connectivity of ventral striatum (Dandash et al, 2014), striatonigral disconnection is a viable mechanism for targeted treatment of refractory schizophrenia. This work marks a potentially important bridge toward dealing with this chronically incapacitating aspect of the illness.

\section{FUNDING AND DISCLOSURE}

The authors declare no conflict of interest.

\section{ACKNOWLEDGMENTS}

We thank all volunteers for their participation in the study. This work was supported by a Medical Research Council New Investigator award and a European Research Council Consolidator Award to SSS, and developed by the National Institute for Health Research (NIHR) Mental Health Biomedical Research Centre at South London and Maudsley NHS Foundation Trust and King's College London, and a joint infrastructure grant from Guy's and St Thomas' Charity and the Maudsley Charity.

\section{REFERENCES}

Alexander GE, DeLong MR, Strick PL (1986). Parallel organization of functionally segregated circuits linking basal ganglia and cortex. Annu Rev Neurosci 9: 357-381.

Backasch B, Sommer J, Klohn-Saghatolislam F, Muller MJ, Kircher TT, Leube DT (2014). Dysconnectivity of the inferior frontal gyrus: implications for an impaired self-other distinction in patients with schizophrenia. Psychiatry Res 223: 202-209.

Belin D, Everitt BJ (2008). Cocaine seeking habits depend upon dopamine-dependent serial connectivity linking the ventral with the dorsal striatum. Neuron 57: 432-441.

Botvinick MM, Huffstetler S, McGuire JT (2009). Effort discounting in human nucleus accumbens. Cogn Affect Behav Neurosci 9: 16-27.

Braver TS, Barch DM, Cohen JD (1999a). Cognition and control in schizophrenia: a computational model of dopamine and prefrontal function. Biol Psychiatry 46: 312-328.

Braver TS, Cohen JD (1999b). Dopamine, cognitive control, and schizophrenia: the gating model. Prog Brain Rese 121: 327-349.

Conley RR, Kelly DL (2001). Management of treatment resistance in schizophrenia. Biol Psychiatry 50: 898-911. 
Creese I, Burt DR, Snyder SH (1976). Dopamine receptor binding predicts clinical and pharmacological potencies of antischizophrenic drugs. Science 192: 481-483.

Croxson PL, Walton ME, O'Reilly JX, Behrens TE, Rushworth MF (2009). Effort-based cost-benefit valuation and the human brain. J Neurosci 29: 4531-4541.

D'Ardenne K, Eshel N, Luka J, Lenartowicz A, Nystrom LE, Cohen JD (2012). Role of prefrontal cortex and the midbrain dopamine system in working memory updating. Proc Natl Acad Sci USA 109: 19900-19909.

Dandash O, Fornito A, Lee J, Keefe RS, Chee MW, Adcock RA et al (2014). Altered striatal functional connectivity in subjects with an at-risk mental state for psychosis. Schizophr Bull 40: 904-913.

Demjaha A, Egerton A, Murray RM, Kapur S, Howes OD, Stone JM et al (2014). Antipsychotic treatment resistance in schizophrenia associated with elevated glutamate levels but normal dopamine function. Biol Psychiatry 75: e11-e13.

Demjaha A, Murray RM, McGuire PK, Kapur S, Howes OD (2012). Dopamine synthesis capacity in patients with treatment-resistant schizophrenia. Am J Psychiatry 169: 1203-1210.

Di Martino A, Scheres A, Margulies DS, Kelly AM, Uddin LQ, Shehzad Z et al (2008). Functional connectivity of human striatum: a resting state FMRI study. Cereb Cortex 18: 2735-2747.

Diaconescu AO, Jensen J, Wang $H$, Willeit $M$, Menon $M$, Kapur S et al (2011). Aberrant effective connectivity in schizophrenia patients during appetitive conditioning. Front Hum Neurosci 4: 239.

Dratcu L, Grandison A, McKay G, Bamidele A, Vasudevan V (2007). Clozapine-resistant psychosis, smoking, and caffeine: managing the neglected effects of substances that our patients consume every day. Am J Ther 14: 314-318.

Fornito A, Harrison BJ, Goodby E, Dean A, Ooi C, Nathan PJ et al (2013). Functional dysconnectivity of corticostriatal circuitry as a risk phenotype for psychosis. JAMA Psychiatry 70: 1143-1151.

Fox MD, Snyder AZ, Vincent JL, Corbetta M, Van Essen DC, Raichle ME (2005). The human brain is intrinsically organized into dynamic, anticorrelated functional networks. Proc Natl Acad Sci USA 102: 9673-9678.

Frank J, Lang M, Witt SH, Strohmaier J, Rujescu D, Cichon S et al (2015). Identification of increased genetic risk scores for schizophrenia in treatment-resistant patients. Mol Psychiatry 20: 913.

Fusar-Poli P, Meyer-Lindenberg A (2013). Striatal presynaptic dopamine in schizophrenia, part II: meta-analysis of [(18)F/(11) C]-DOPA PET studies. Schizophr Bull 39: 33-42.

Ginovart N, Wilson AA, Hussey D, Houle S, Kapur S (2009). D2-receptor upregulation is dependent upon temporal course of D2-occupancy: a longitudinal [11C]-raclopride PET study in cats. Neuropsychopharmacology 34: 662-671.

Gonzalez-Rodriguez A, Molina-Andreu O, Imaz Gurrutxaga ML, Catalan Campos R, Arroyo MB (2014). A descriptive retrospective study of the treatment and outpatient service use in a clinical group of delusional disorder patients. Rev Psiquiatr Salud Ment 7: 64-71.

Haber SN, Fudge JL (1997). The interface between dopamine neurons and the amygdala: implications for schizophrenia. Schizophr Bull 23: 471-482.

Haber SN, Fudge JL, McFarland NR (2000). Striatonigrostriatal pathways in primates form an ascending spiral from the shell to the dorsolateral striatum. J Neurosci 20: 2369-2382.

Haber SN, Knutson B (2010). The reward circuit: linking primate anatomy and human imaging. Neuropsychopharmacology 35: $4-26$.

Hassan AN, De Luca V (2015). The effect of lifetime adversities on resistance to antipsychotic treatment in schizophrenia patients. Schizophr Res 161: 496-500.
He Z, Deng W, Li M, Chen Z, Jiang L, Wang Q et al (2013). Aberrant intrinsic brain activity and cognitive deficit in firstepisode treatment-naive patients with schizophrenia. Psychol Med 43: 769-780.

Heinz A, Schlagenhauf F (2010). Dopaminergic dysfunction in schizophrenia: salience attribution revisited. Schizophr Bull 36: 472-485.

Howes OD, Montgomery AJ, Asselin MC, Murray RM, Valli I, Tabraham $\mathrm{P}$ et al (2009). Elevated striatal dopamine function linked to prodromal signs of schizophrenia. Arch Gen Psychiatry 66: $13-20$.

Insel TR (2014). The NIMH Research Domain Criteria (RDoC) Project: precision medicine for psychiatry. Am J Psychiatry 171: 395-397.

Kapur S, Mizrahi R, Li M (2005). From dopamine to salience to psychosis-linking biology, pharmacology and phenomenology of psychosis. Schizophr Res 79: 59-68.

Kay SR, Fiszbein A, Opler LA (1987). The positive and negative syndrome scale (PANSS) for schizophrenia. Schizophr Bull 13: 261-276.

Kegeles LS, Abi-Dargham A, Frankle WG, Gil R, Cooper TB, Slifstein $\mathrm{M}$ et al (2010). Increased synaptic dopamine function in associative regions of the striatum in schizophrenia. Arch Gen Psychiatry 67: 231-239.

Knutson B, Cooper JC (2005). Functional magnetic resonance imaging of reward prediction. Curr Opin Neurol 18: 411-417.

Kolakowska T, Williams AO, Jambor K, Ardern M (1985). Schizophrenia with good and poor outcome. III: Neurological 'soft' signs, cognitive impairment and their clinical significance. Br J Psychiatry 146: 348-357.

Leh SE, Ptito A, Chakravarty MM, Strafella AP (2007). Fronto-striatal connections in the human brain: a probabilistic diffusion tractography study. Neurosci Lett 419: 113-118.

Lehericy S, Ducros M, Van de Moortele PF, Francois C, Thivard L, Poupon C et al (2004). Diffusion tensor fiber tracking shows distinct corticostriatal circuits in humans. Ann Neurol 55: 522-529.

Lieberman JA, Stroup TS, McEvoy JP, Swartz MS, Rosenheck RA, Perkins DO et al (2005). Effectiveness of antipsychotic drugs in patients with chronic schizophrenia. $N$ Engl J Med 353: 1209-1223.

Lynall ME, Bassett DS, Kerwin R, McKenna PJ, Kitzbichler M, Muller U et al (2010). Functional connectivity and brain networks in schizophrenia. J Neurosci 30: 9477-9487.

Mechelli A, Riecher-Rossler A, Meisenzahl EM, Tognin S, Wood SJ, Borgwardt SJ et al (2011). Neuroanatomical abnormalities that predate the onset of psychosis: a multicenter study. Arch Gen Psychiatry 68: 489-495.

Moghaddam B, Adams B, Verma A, Daly D (1997). Activation of glutamatergic neurotransmission by ketamine: a novel step in the pathway from NMDA receptor blockade to dopaminergic and cognitive disruptions associated with the prefrontal cortex. J Neurosci 17: 2921-2927.

Nakajima S, Takeuchi H, Plitman E, Fervaha G, Gerretsen P, Caravaggio $\mathrm{F}$ et al (2015). Neuroimaging findings in treatmentresistant schizophrenia: a systematic review: lack of neuroimaging correlates of treatment-resistant schizophrenia. Schizophr Res 164: 164-175.

Nauta WJH, Domesick VB. Crossroads of limbic and striatal circuitry: hypothalamic-nigral connections. In: Livingston KE, Hornykiewicz O (eds). Limbic Mechanisms. Plenum Publishing Corp.: New York, 1978, pp 75-93.

O'Doherty J, Dayan P, Schultz J, Deichmann R, Friston K, Dolan RJ (2004). Dissociable roles of ventral and dorsal striatum in instrumental conditioning. Science 304: 452-454.

Papanastasiou E, Stone JM, Shergill S (2013). When the drugs don't work: the potential of glutamatergic antipsychotics in schizophrenia. Br J Psychiatry 202: 91-93. 
Quide Y, Morris RW, Shepherd AM, Rowland JE, Green MJ (2013). Task-related fronto-striatal functional connectivity during working memory performance in schizophrenia. Schizophr Res 150: 468-475.

Raichle ME, MacLeod AM, Snyder AZ, Powers WJ, Gusnard DA, Shulman GL (2001). A default mode of brain function. Proc Natl Acad Sci USA 98: 676-682.

Rose D, Pevalin DJ (2001). The national statistics socio-economic classification: unifying official and sociological approaches. ISER Working Papers. Paper 2001-4. University of Essex: Colchester.

Samaha AN, Seeman P, Stewart J, Rajabi H, Kapur S (2007). 'Breakthrough' dopamine supersensitivity during ongoing antipsychotic treatment leads to treatment failure over time. J Neurosci 27: 2979-2986.

Sarpal DK, Robinson DG, Lencz T, Argyelan M, Ikuta T, Karlsgodt K et al (2015). Antipsychotic treatment and functional connectivity of the striatum in first-episode schizophrenia. JAMA Psychiatry 72: 5-13.

Seeman P, Lee T (1975). Antipsychotic drugs: direct correlation between clinical potency and presynaptic action on dopamine neurons. Science 188: 1217-1219.

Somogyi P, Bolam JP, Totterdell S, Smith AD (1981). Monosynaptic input from the nucleus accumbens-ventral striatum region to retrogradely labelled nigrostriatal neurones. Brain Res 217: 245-263.

Suckling J (2011). Correlated covariates in ANCOVA cannot adjust for pre-existing differences between groups. Schizophr Res 126: 310-311.
Tomasi D, Volkow ND (2014). Mapping small-world properties through development in the human brain: disruption in schizophrenia. PLoS One 9: e96176.

Van Dijk KR, Sabuncu MR, Buckner RL (2012). The influence of head motion on intrinsic functional connectivity MRI. Neuroimage 59: 431-438.

Wechsler D (1999). Wechsler Abbreviated Scale of Intelligence. The Psychological Corporation: Harcourt Brace \& Company: New York.

White TP, Gilleen J, Shergill SS (2013). Dysregulated but not decreased salience network activity in schizophrenia. Front Hum Neurosci 7: 65.

Whitfield-Gabrieli S, Nieto-Castanon A (2012). Conn: a functional connectivity toolbox for correlated and anticorrelated brain networks. Brain Connect 2: 125-141.

Wolkin A, Brodie JD, Barouche F, Rotrosen J, Wolf AP, Smith M et al (1989). Dopamine receptor occupancy and plasma haloperidol levels. Arch Gen Psychiatry 46: 482-484.

Yoon JH, Westphal AJ, Minzenberg MJ, Niendam T, Ragland JD, Lesh $\mathrm{T}$ et al (2014). Task-evoked substantia nigra hyperactivity associated with prefrontal hypofunction, prefrontonigral disconnectivity and nigrostriatal connectivity predicting psychosis severity in medication naïve first episode schizophrenia. Schizophr Res 159: 521-526.

Yoon JH, Minzenberg MJ, Raouf S, D'Esposito M, Carter CS (2013). Impaired prefrontal-basal ganglia functional connectivity and substantia nigra hyperactivity in schizophrenia. Biol Psychiatry 74: 122-129.

Supplementary Information accompanies the paper on the Neuropsychopharmacology website (http://www.nature.com/npp) 